REDES- Revista hispana para el análisis de redes sociales

Vol.16,\#5, Junio 2009

http://revista-redes.rediris.es

\title{
La invitación al matrimonio. Una aproximación a las redes de sociabilidad de la pareja
}

\begin{abstract}
Florence Maillochon, Centre Maurice Halbwachs - ERIS (France) ${ }^{1}$
\section{Resumen}

Este artículo describe lo que está en juego en la elaboración de la lista de invitados a las ceremonias de matrimonio. Esta circunstancia ofrece al sociólogo una oportunidad excepcional para observar una "red de sociabilidad" objetivada por los actores mismos. Al poner en relación el contenido concreto de estas listas con los discursos de las parejas sobre las lógicas y las dudas que preceden a la selección, permite matizar el carácter subtancialista que se atribuye generalmente a las "redes". Invitar a alguien al propio matrimonio no es una simple enumeracion/mirada de sus relaciones amicales, sino una acción performativa que permite construirlas y proyectarlas al futuro. La invitación al matrimonio parece también fuertemente condicionada por las posiciones sociales respectivas de las personas involucradas, de manera tal que el reconocimiento afectivo y el reconocimiento social resultan a menudo indisociables en la elaboración de esta relación. Los invitados al matrimonio no representan solamente un estado de hecho de la "red amical", producto de la historia de la pareja e inscrito en el pasado de cada uno, sino también una promesa sobre la posible evolución de la proyección afectiva, y también social, de dicha pareja.
\end{abstract}

Palabras clave: Matrimonio, relaciones, amistad, reconocimiento.

\section{Résumé}

Cet article décrit les enjeux sociaux de l'élaboration de la liste d'invités aux cérémonies de mariage. Cette circonstance constitue une opportunité rare pour le sociologue d'observer un « réseau de sociabilité » objectivé par les acteurs euxmêmes. La mise en regard du contenu effectif de ces listes avec les discours recueillis auprès des couples sur les logiques et les hésitations ayant présidé à leurs choix permet de nuancer le caractère substantialiste qui est souvent attribué aux « réseaux ». Inviter quelqu'un à son mariage n'est pas une simple opération de mise à plat de ses relations amicales, mais une action performatrice qui permet de les construire et de les projeter dans l'avenir. Cet acte est aussi largement conditionné par les positions sociales respectives des personnes en jeu de sorte que reconnaissance affective et reconnaissance sociale y apparaissent souvent indissociables. L'ensemble des invités au mariage ne représente donc pas seulement un état de fait du réseau amical du couple, mais aussi une promesse d'évolution de son rayonnement affectif et social.

Mots-clés: Mariage, liens, amitié, reconnaissance.

\begin{abstract}
This article describes the social factors involved in drawing up a list of wedding guests. This circumstance provides a rare opportunity for sociologists to observe a "social network" objectified by the actors themselves. Comparing the actual content of these lists with the couples' accounts of the reasons for their choices and for their hesitations provides a means to qualify the reputedly substantialist nature of social networks. A guest list is not a simple inventory of friendships; inviting someone to one's wedding is a performative action enabling the couple to contruct relationships and project them into the future. Choices are also strongly conditioned
\end{abstract}

\footnotetext{
${ }^{1}$ florence.maillochon@ens.fr. Traducción: Ana Masullo (Centre Maurice Halbwachs - ERIS).
} 
by the respective social positions of the persons concerned, such that affective recognition and social recognition often become indissociable. Hence, the list of wedding guests is not simply a photograph of the couple's network of friends, but also a promise of future affective and social fulfilment.

Key words: Marriage, friendship, recognition.

El momento en que dos personas se unen para toda la vida es también un momento de reflexión sobre las relaciones que han establecido antes, juntas o separadamente: aquellas a las que consideran importante invitar para que los acompañen en ese día especial. A pesar de la importancia de esta selección, los invitados a la boda jamás han sido objeto de estudios específicos, con dos excepciones: la biografía sobre "el doble matrimonio de Jean Célesse", realizada por Yvette Delsaut (1976), donde se analiza con detalle los problemas del encuentro de dos mundos familiares, socialmente diferentes, y la descripción de los nuevos rituales realizada por Martin Ségalen (1995, 1998), que se refiere a las transformaciones de los roles de los amigos de la pareja en la boda. Este artículo propone una descripción de las ceremonias de matrimonio de jóvenes adultos en Francia bajo el ángulo de las dinámicas relacionales que se ponen en juego. La ceremonia matrimonial proporciona al sociólogo una oportunidad poco común de observar detalladamente una "red" de sociabilidad. En efecto, la lista de invitados que es una de las decisiones más importantes en los preparativos del matrimonio, con independencia del interés que pueda prestarle el investigador ${ }^{2}$ : puede considerarse como una red parcial, pero también parcializada, de dos individuos que proyectan casarse. Se elabora de hecho, bajo diferentes condiciones que limitan y redefinen sus contornos: limitaciones estructurales relacionadas con el tamaño de las familias de origen de los esposos y la pertenencia de los cónyuges a diferentes círculos sociales; restricciones formales debidas a la exigencia de un cierto fasto que rodea casi sistemáticamente las fiestas matrimoniales: limitaciones financieras impuestas por el presupuesto de los novios y de sus familias, siempre escasos en comparación con sus aspiraciones.

El enunciado de estas condiciones diferentes permite esbozar el contexto en que se elabora la lista de invitados, pero no basta para describir con detalle el círculo de

\footnotetext{
2 Uno de los problemas principales de los análisis de las redes de sociabilidad es efectivamente la reunión de informaciones y la elección de un buen "generador de nombres" (Gribaudi, 1999; Bidart, Charbonneau, 2007) que permita identificar una o varias dimensiones interesantes. En este caso particular, la lista de invitados es una lista de datos relacionales y no el producto de un dispositivo de investigación ad hoc. Solo las informaciones recogidas para describir estos invitados y sus relaciones con la pareja están determinadas por el/la investigador/a.
} 
íntimos que finalmente acompaña a los esposos el día de su matrimonio, lo que representa para ellos y lo que representa socialmente. Nuestra hipótesis es que la lista de invitados no es sólo el registro de una red afectiva asociada al pasado, sino también una estimación de su evolución ulterior y del reconocimiento social que promete o augura para el futuro.

Esta red no puede comprenderse (ni en sus límites, ni en su significación) sin explorar la relación "de invitación" que le da sentido y consistencia. Con este fin, se emplearán y compararán dos tipos de datos para tratar de describir el conjunto de los acuerdos, las elecciones y presiones, que las parejas se ven llevadas a efectuar: por otra parte, la descripción exhaustiva del grupo de invitados ${ }^{3}$ de acuerdo con sus características sociodemográficas y relacionales; por otra parte, los relatos de las parejas donde exponen sus procedimientos de selección, sus vacilaciones y sus dudas, así como su percepción de sus elecciones concretas (cf. Metodologia). Es, en efecto, en este ir y venir permanente entre dos tipos de aclaraciones de la lista de invitados - y sus eventuales contradicciones- que se percibe mejor el carácter socialmente construido de la invitación al matrimonio. Invitar a una persona al propio matrimonio no es un acto anodino, aun cuando las parejas suelen disimular la complejidad real de la tarea al referirse a ella. Así, explorando igualmente lo que representa la decisión opuesta, "no invitar a alguien", o su simétrico "ser invitado", se percibe mejor la significación de tal acto y sus implicaciones no sólo personales y relacionales, sino también sociales.

\section{Metodología}

Los datos analizados en este artículo provienen de una investigación en desarrollo sobre las ceremonias del matrimonio entre jóvenes franceses.

Se estudiaron 25 casos de "primer matrimonio". A fin de limitar las variaciones ligadas a las particularidades biográficas de los ciclos de vida, las parejas incluidas tienen entre 24 y 33 años. La muestra se diseñó con la finalidad de mantener una importante diversidad social y geográfica. Los individuos interrogados ocupan posiciones sociales muy diferentes (obrero, abogado, empleado de banco, profesor de escuela secundaria, manager, etc.), habitantes de París o la región parisiense, de Normandía (Caen y las comunidades rurales circundantes). Se recogió una variedad de tipos de datos para cada pareja: dos entrevistas semidirigidas, realizadas antes y después del matrimonio, cuestionarios biográficos sintéticos sobre la historia individual y familiar, cuadros del conjunto de los invitados (así como fotos y videos de las fiestas de casamiento, no incluidos en este artículo).

\footnotetext{
${ }^{3}$ Este artículo se concentra en el análisis de las invitaciones a amigos, dejando de lado las familiares (estudiadas en Maillochon 2002, 2008a) que se plantean en términos ligeramente diferentes, y requieren en particular tomar en cuenta la estructura y el tamaño de las familias de los miembros de la pareja que presentan muchas variaciones, así como las negociaciones con los padres.
} 
El cuadro recapitulativo de los invitados permitió reunir información sobre sus características sociodemográficas (sexo, edad, profesión, lugar de residencia), sobre el origen y la antigüedad de su vínculo con cada uno de los esposos así como sobre la naturaleza actual de esta relación, sobre su tipo y sobre su frecuencia de interacción).

\section{La red expresiva y afectiva: la invitación como expresión de una relación amical, pasada y futura.}

\subsection{La invitación, una "relación" social bajo presión}

En la preocupación por hacer una boda "que los represente" - una de las exigencias principales del matrimonio contemporáneo (Maillochon, 2008b) - los esposos dan mucha importancia a la selección de sus convidados y a que esta elección sea vista como algo personal y/o conyugal. Esta reivindicación es causa de numerosas discusiones con los padres para no dejarse imponer una lista familiar o demasiado tradicional (Castrén y Maillochon, 2009). Ello también se refleja en la debilidad del derecho de los padres a fiscalizar a los invitados, aunque se los considere desde esta etapa como actores clave de la fiesta (Segalen, 1995).

En esa situación, a menudo los novios presentan su lista de invitados como una elección libre, sin limitaciones familiares, y como obvia, una elección por amistad. Invitan a los "íntimos", los "ineludibles", de los que no podrían prescindir. El matrimonio es la ocasión para reunir a su alrededor, a todas las personas que se aman, todas las que cuentan. Como afirman Christelle (empleada) y Sébastien (responsable de compras), la lista se impone por sí misma.

Christelle: “Sí, es muy fácil. Yo hago mi lista a mano alzada, así...

Sébastien: Muy fácil."

Como la amistad que expresa, la invitación al matrimonio no daría lugar a la duda o al cálculo, que de cierta manera negarían esa amistad. Noemie (abogada) lo expone de este modo:

Noemie: "En definitiva, las personas que hemos invitado son aquellas que es evidente que teníamos que invitar y, si dudábamos, nos decíamos: 'en realidad no es importante para nosotros que vengan o no vengan', como suele decirse: 'si tenemos dudas es porque no nos importa que estén o no estén'".

La elección de los invitados, como la de los amigos, se presenta siempre como una certidumbre y una libre elección, que de alguna manera resiste a toda explicación, 
a toda justificación. El principio generador de la lista, parece así hacer eco a la fórmula de Montaigne: "Porque era él, porque era yo", cuya persistencia en las representaciones sociales de la amistad ha sido ilustrada por Bidart (1991). Describe igualmente cuan distanciadas pueden estar estas representaciones de las relaciones concretamente vividas y cómo estas relaciones se constituyen.

$\mathrm{Si}$, al igual que los amigos, los invitados aparecen como una necesidad, es por negligencia u omisión del proceso a menudo complejo que ha permitido llegar a ese resultado y eliminar, de hecho, otros procesos posibles. Sin embargo, es gracias a todas esas operaciones sociales cuyo producto -amigos invitados- aparece como obvio que nos parece interesante dar claridad sociológica al respecto. La invitación a la boda no puede corresponder a restricciones sociales que proyectarían una sombra de duda sobre la intensidad de la relación o la amistad que revela; no obstante, como la amistad, se presta a diversas limitaciones y estrategias, en la medida en que es también la invitación a un hecho social ${ }^{4}$.

En efecto, aun cuando el matrimonio es percibido y reivindicado cada vez más como la oportunidad de hacer la (gran) fiesta, es una forma completamente específica que poco admite el registro de lo informal o lo imprevisto (Segalen, 1997 ; Mailochon, 2008a). Previsto desde hace tiempo, preparado con gran cuidado durante un largo período de tiempo y con una lista de convidados limitada (donde los invitados sorpresa no son siempre bienvenidos y los invitados de invitados son inimaginables ${ }^{5}$ ), se distingue también de todos los demás tipos de celebración que una pareja joven puede organizar para reunir a sus amigos. Por su misma naturaleza, la boda no es una simple fiesta de amigos, aun cuando los esposos insistan decididamente en ello, para escapar también al formalismo convencional y tradicional del matrimonio. La lista de invitados no expresa solamente un vínculo que une a cada convidado con los futuros esposos, sino también la naturaleza misma del evento que estos desean crear. En la mayoría de los casos, la pareja presenta la selección de invitados y del tipo de boda como dos hechos independientes, apoyada en esto por la mayor parte de las guías de organización de matrimonios y los sitios de internet de ayuda a su preparación ${ }^{6}$.

\footnotetext{
${ }^{4}$ Por lo tanto, permite formar una red que, en la mayoría de los casos (Granovetter, 1982), no es ni simplemente afectiva ni totalmente instrumental.

${ }^{5}$ Con una excepción, el/la esposo/a del amigo era aún desconocida por los consortes. Se trata del único caso de invitación "transitiva" previsible en la lista de los convidados amigos.

6 Dos ejemplos de la cronología de las tareas a realizar en la preparación del matrimonio según dos sitios frecuentemente visitados.
} 
Los relatos de los preparativos de la boda, y de los innumerables cuestionamientos que plantea, muestran de manera clara la imposibilidad de pensar independientemente la elección del tipo de festejo y la selección de los invitados, en la que cada una condiciona a la otra. La elección misma del salón de la recepción (pequeño o grande, sencillo o majestuoso) constituye un buen ejemplo. La lista de invitados no es sólo la expresión de los "ineludibles" por amistad, sino también de una selección acorde con la búsqueda de un cierto efecto en la celebración de un evento social. No se trata solamente de la naturaleza del vínculo, sino del contexto de la celebración que sirve de soporte a la invitación a la boda.

\subsection{La invitación, una relación polimorfa}

Aun cuando todas las parejas declaren que no desean reunirse sino con los íntimos - los "ineludibles" - a menudo les resulta difícil definir el sentido de esta relación con mayor precisión que afirmando que es obvio. Definir un vínculo no es tarea fácil, ni para las parejas que tratan de terminar su lista, ni para los sociólogos que se proponen objetivar esta descripción (Allan, 1979 ; Fischer, 1982 ; Bidart, 1987). Según algunas parejas, cada amigo es invitado por una razón diferente, como en el caso de Marianne (ingeniero y estudiante en doctorado) y Cédric (responsable de ventas), que, a pesar de tener un gran número de convidados, insisten en la especificidad de cada uno de los vínculos. Según otras, los amigos forman un grupo indistinto, donde todo el mundo tiene la misma importancia, negándose a hacer una diferenciación, y menos aún una jerarquización.

La intensidad y/o la familiaridad de la relación, igual que la frecuencia de las interacciones, son los dos criterios principales de elección enunciados por las parejas. Marion (responsable de logística) y Cyrille (asistente) lo ilustran.

\section{http://www. aufeminin.com}

Organizar el encuentro de los padres

Definir el estilo y el presupuesto del casamiento

Contactar el ayuntamiento (y quizás el cura, el rabino, el imán...) para elegir la fecha, la hora y el lugar de la ceremonia

Encontrar rápidamente el lugar de la recepción (¡Rápidamente, ya que los mejores lugares a menudo son contratados con un año de anticipación!)

Comenzar a escoger el vestido de la novia

Hacer la lista de invitados

http://www.organisation-mariage.net/planning-du-mariage.htm

Entre las 61 tareas que deben realizarse en 12 meses, la elaboración de una lista provisoria de invitados está en noveno lugar, después de la elección del salón de recepción (segundo), la definición de un presupuesto (séptimo) y la elección del automóvil (octavo). Siguiendo la lógica cartesiana de descomponer un problema en subproblemas, los consejos dejan de lado una dificultad importante de la realización de este trabajo complejo: las interacciones y retroacciones de cada una de estas operaciones. 
Marion: "Era claro que había personas a las que queríamos ver en nuestra boda y otras que no debían estar. La gente con la que nos vemos con frecuencia debe estar en el vino de honor?

Cyrille: No queremos invitar a gente que vemos una vez al año. Eso no nos interesa".

De todos modos, estas dos posiciones son difíciles de objetivar y pueden presentarse de diversas maneras. En esta investigación, la intensidad del vínculo con cada uno de los invitados fue calificada según una escala, graduada de 1 (el más fuerte) a 4 (el menos fuerte) ${ }^{8}$, en la cual los esposos hacían su elección. La frecuencia de interacción se indexaba de acuerdo con la fecha de su último encuentro ${ }^{9}$.

Calificada de este modo, la proximidad de los amigos invitados a los matrimonios, tal como aparece en el análisis cuantitativo de las listas de convidados, resulta más matizada que en las declaraciones de los esposos, diferencia que es un testimonio verosímil de las estrategias que desplegaron para adaptarse a las limitaciones y para evitarlas.

En efecto, como se ve en la Tabla 1, pareciera que los invitados al matrimonio no son todos amigos apreciados, en particular en el caso de las esposas, que son, en promedio, más severas en sus apreciaciones que los esposos. Las relaciones más fuertes (codificadas 1 ) no representan sino una parte reducida de los invitados, menos de la quinta parte en promedio y para la mitad de las parejas ${ }^{10}$, menos del $11 \%$ para las mujeres y menos del $17 \%$ para sus maridos). De manera general, la participación de los amigos más próximos (codificados 1 ó 2 ) es casi equivalente a la de los que no son tan próximos (codificados 3 ó 4) y para la mitad de las parejas son claramente menos numerosos (mediana de $41 \%$ para las mujeres, y de $47 \%$ para los hombres). Sobre todo, el número de invitados que los esposos no

\footnotetext{
${ }^{7}$ En Francia, después de la ceremonia religiosa o civil, se acostumbra invitar a los presentes a un coktail, pero solo algunos de estos últimos están invitados a la cena o celebración que se realiza posteriormente.

8 La encuesta incluía otro indicador (no analizado en este artículo): la naturaleza del vínculo (dejada a la libre apreciación de los esposos) y que, de hecho, es poco descriptiva. Las anotaciones hacen especialmente referencia a apreciaciones vagas, como "amistosa", "conocimiento", "colega".

9 El cuestionario incluyó otros indicadores de interacción no considerados aquí. Conciernen la naturaleza de diversas actividades realizadas en grupo en el curso del año: ya sea en el domicilio propio, ya sea en el domicilio del otro, o en otro lugar.

10 Teniendo en cuenta el número reducido de las parejas que respondieron a estas preguntas, el promedio es un indicador poco sólido que se emplea sólo de manera ilustrativa. Es preferible la media, que da una medida de la dispersión y permite evitar la distorsión introducida por el promedio por la inclusión de dos parejas Cat-Ala y Sop-Vin, que no invitaron a más de dos amigos.
} 
consideran totalmente próximos (codificados 4) no es nada despreciable. Para este fenómeno se encuentran numerosas explicaciones estructurales ligadas principalmente a la diferencia de los vínculos que cada uno de los esposos puede mantener con la misma persona ${ }^{11}$. El esposo y la esposa no aprecian forzosamente a los amigos respectivos de la misma manera; es el caso de Noémie (abogada) que experimenta una cierta incomodidad ante un amigo de trabajo de Vincent (diseñador gráfico) cuando éste actúa negativamente con una antigua compañera de su mujer. Un miembro de la pareja puede invitar personas con las que el otro jamás se ha encontrado, caso poco frecuente (Tabla 2) pero que de todos modos aparece en el caso de Fabien (ingeniero), quien invita a compañeros de infancia o de deportes que Florence (empleada) no conoce, puesto que prefiere verse con ellos solo. O el caso de de Benoit (administrador en informática), que reúne compañeros de juego de roles que Valerie (asistente técnica de laboratorio) ha tenido poca ocasión de frecuentar.

\begin{tabular}{|c|c|c|c|c|c|c|c|}
\hline \multirow{2}{*}{$\begin{array}{c}\text { Nombre de la } \\
\text { pareja }\end{array}$} & \multicolumn{2}{|c|}{ Índice = 1} & \multicolumn{2}{|c|}{ Índice $<=2$} & \multicolumn{2}{|c|}{ Índice $=4$} & \multirow{2}{*}{$\begin{array}{c}\mathbf{N} \\
\text { amigos }\end{array}$} \\
\hline & $\begin{array}{c}\text { Por la } \\
\text { esposa }\end{array}$ & $\begin{array}{c}\text { Por el } \\
\text { esposo }\end{array}$ & $\begin{array}{l}\text { Por la } \\
\text { esposa }\end{array}$ & $\begin{array}{l}\text { Por el } \\
\text { esposo }\end{array}$ & $\begin{array}{c}\text { Por la } \\
\text { esposa }\end{array}$ & $\begin{array}{c}\text { Por el } \\
\text { esposo }\end{array}$ & \\
\hline cat ala & 100 & 100 & 100 & 100 & 0 & 0 & 2 \\
\hline sop vin & 0 & 0 & 100 & 100 & 0 & 0 & 2 \\
\hline sid chr & 0 & 10 & 20 & 30 & 40 & 30 & 9 \\
\hline nat thi & 0 & 28 & 21 & 50 & 28 & 0 & 14 \\
\hline mar cyr & 13 & 17 & 73 & 58 & 13 & 11 & 17 \\
\hline san ste & 23 & 12 & 29 & 24 & 0 & 0 & 17 \\
\hline flo fab & 23 & 23 & 41 & 53 & 12 & 35 & 19 \\
\hline ell emm & 47 & 19 & 24 & 43 & 14 & 5 & 21 \\
\hline del pas & 9 & 23 & 63 & 32 & 4 & 0 & 22 \\
\hline val ben & 14 & 17 & 41 & 62 & 10 & 0 & 29 \\
\hline gae pie & 11 & 11 & 52 & 44 & 7 & 15 & 29 \\
\hline cel thi & 7 & 3 & 30 & 17 & 37 & 40 & 30 \\
\hline mar ced & 3 & 8 & 90 & 70 & 0 & 4 & 30 \\
\hline chr seb & 2 & 23 & 55 & 44 & 2 & 0 & 47 \\
\hline noe vin & 32 & 30 & 32 & 47 & 8 & 4 & 49 \\
\hline Media & 19 & 22 & 51 & 52 & 12 & 10 & 22 \\
\hline Mediana & 11 & 17 & 41 & 47 & 8 & 4 & 21 \\
\hline
\end{tabular}

Tabla 1. Índice de proximidad de acuerdo a cada amistad invitada a la boda (\%).

Leyenda: En relación a los 49 amigos invitados por la pareja Noe-Vin, Noémie considera el $32 \%$ como muy próximos y el $8 \%$ como lejanos (respectivamente el $30 \%$ próximos y el $4 \%$ de lejanos para Vincent).

\footnotetext{
${ }^{11}$ Esta investigación se funda de hecho en la recolección de las opiniones de las esposas y esposos sobre cada invitado a fin de no considerar desde el comienzo la pareja como un espacio de decisión colectiva sino de mostrar, por el contrario, los ajustes sobre los cuales esta relación se apoya (Maillochon 2008c).
} 
Nota: La comparación de la red social centrada egocentrada no es una tarea fácil porque requiere la comparación de muy diferentes espacios. Su análisis cuantitativo requiere la descripción de cada una de las redes de acuerdo a un criterio (el \% de personas de la misma PEC que el marido, por ejemplo) y la comparación de los índices obtenidos para cada pareja. 


\begin{tabular}{|c|c|c|c|c|c|}
\hline \multirow{2}{*}{$\begin{array}{c}\text { Nombre de la } \\
\text { pareja }\end{array}$} & \multicolumn{2}{|c|}{ Nunca vistos (N) } & \multicolumn{2}{|c|}{ Más de un año sin verlos (\%) } & \multirow[b]{2}{*}{$\mathbf{N}$ amigos } \\
\hline & $\begin{array}{c}\text { Por la } \\
\text { esposa }\end{array}$ & $\begin{array}{c}\text { Por el } \\
\text { esposo }\end{array}$ & Por la esposa & Por el esposo & \\
\hline cat ala & & & 0 & 0 & 2 \\
\hline sop vin & & & 0 & 0 & 2 \\
\hline sid chr & & & 0 & 0 & 9 \\
\hline nat thi & & & 0 & 0 & 14 \\
\hline mar cyr & & & 22 & 0 & 17 \\
\hline flo fab & & 1 & 0 & 2 & 19 \\
\hline ell emm & & & 15 & 4 & 21 \\
\hline del pas & 1 & & 5 & 9 & 22 \\
\hline val ben & 5 & 1 & 14 & 10 & 29 \\
\hline gae pie & 1 & 3 & 11 & 11 & 29 \\
\hline cel thi & & & 20 & 11 & 30 \\
\hline mar ced & & 1 & 7 & 14 & 30 \\
\hline chr seb & & & 24 & 24 & 47 \\
\hline noe vin & 2 & 1 & 7 & 30 & 49 \\
\hline san ste & & & 24 & 35 & 17 \\
\hline Media & & & 9,9 & 10 & 22 \\
\hline Mediana & & & 7 & 9 & 21 \\
\hline
\end{tabular}

Tabla 2. Frecuentación de cada amigo invitado a la boda.

Leyenda: De los 49 amigos invitados por la pareja Noe-Vin, Noémie no conoce a dos personas (respectivamente una para el caso de Vincent); ella no ha visto al $7 \%$ en más de un año (resp. $30 \%$ en el caso de Vincent).

La presencia significativa de invitados con relaciones debiles sugiere que los "ineludibles" no son todos elegidos con el corazón. A pesar de su deseo de elegir personal y libremente a sus invitados, los esposos no pueden evitar un cierto número de presiones ligadas a las convenciones sociales o a la estructura de las relaciones sociales. Junto a los que son "ineludibles" por el afecto que los liga a los esposos, figuran también aquellos a los que no se podría dejar de invitar por razones sociales; un cierto número de personas "ineludibles" porque, por ejemplo, han invitado antes a la pareja a su propio matrimonio y se les debe devolver la invitación. En la lógica del don y del contra-don de Mauss, la invitación "obliga" en el doble sentido de la palabra. De este modo, ciertas personas son invitadas no porque se imponen por ellas mismas, sino porque sus relaciones con otros miembros del entorno de la pareja los imponen casi de hecho. Lo mismo ocurre con determinadas personas invitadas, a pesar de todo, para o por los padres, así como otras que pertenecen a un mismo grupo de amigos al que no se quiere dividir, aun si la pareja no mantiene lazos fuertes con cada uno de ellos. Noémie nos da un buen ejemplo: 
Noémie “(...) hay también personas a las que invitamos porque no quedaba otra alternativa, entre comillas. Gente con la que no se tiene forzosamente nada en común, pero se ha invitado a todas las personas de su entorno, y no se puede dejarlas de lado, porque sabemos que cuando nos han dejado de lado, nos sentimos mal y es una lástima, es que... Yo creo que la regla es más bien ésta: no invitamos a las personas que sabemos que no se sentirán lastimados, ni tristes por no asistir...

Por lo tanto, los amigos invitados están lejos de constituir un grupo afectivo homogéneo e indiferenciado, como se ve igualmente en las diferencias en la frecuencia de las interacciones con cada uno de ellos. Aunque la mayor parte de las parejas tiene encuentros regulares ${ }^{12}$ con los amigos que invitan, todos no se habían frecuentado necesariamente en el curso del año (Tabla 2), o incluso desde hacía varios años. Tal es el caso particular de los esposos con grandes redes de amigos, algunos de cuyos miembros a veces residen lejos de la pareja.

El carácter de obviedad que las parejas dan a la elección de sus invitados se vuelve totalmente relativo apenas empezamos a tratar de precisar esta afirmación. Raros son los invitados que acumulan de hecho todos los índices de proximidad relacional con cada uno de los esposos. Por el contrario, el número nada despreciable de invitados que, en definitiva, se consideran poco próximos o poco frecuentados permite pensar que la elección de los novios está gobernada por otros principios que la simple voluntad de inclusión afectiva de los amigos que los rodean.

\subsection{La fuerza performativa de la invitación}

El acto de invitar a la boda no sólo permite expresar una relación vínculo de amistad como tal; contribuye además a crearla y enaltecerla elevando a los invitados al rango de elegidos, presentes en un evento excepcional. El hecho de compartir este momento privilegiado, reservado a un número limitado, también hace sentir a los invitados la importancia y la amistad que los esposos les brindan.

El acto de invitar no es pues sólo un acto pasivo de inclusión de un número de amigos en su red personal, es también un acto activo de producción o reproducción de su medio amical.

\footnotetext{
12 No se consideran sino los encuentros cara a cara que evidentemente son sólo uno de los medios posibles para mantener y hacer vivir el vínculo. Esta elección se ha hecho con el fin de controlar los efectos de la fractura numérica sobre el ejercicio de la sociabilidad en el seno de ésta, socialmente muy diversa.
} 
Numerosas parejas aprovechan esta ocasión para retomar contacto con personas que han perdido de vista con el tiempo y las obligaciones o por alejamiento geográfico. La invitación permite no sólo recordar la amistad del pasado, expresando la importancia acordada a esta relación, aun cuando se haya debilitado (lo que se materializa en la tarjeta de comunicación del matrimonio o invitación a la boda), sino también volver a abrirle así un posible futuro. La boda, en efecto, reúne a personas que se conocen desde hace tiempo, como se ve en Tabla 3. Casi todas las parejas invitan a personas que conocen desde su infancia, desde hace alrededor de unos veinte años, y la mitad de los invitados se conocen, en promedio, desde hace más de siete años. No debe sorprender entonces que, en estas condiciones, todos los contactos no se hayan mantenido tan vivos como en el ejemplo que nos proporciona Bruno.

Charlotte (politóloga) y Bruno (ingeniero) declaran no haber tenido ningún problema al establecer la lista de sus invitados. Tienen una visión clara de todas las personas indispensables para la celebración y prefieren reducir el precio del menú por persona- que de todas maneras es elevado- antes que privarse de algunos invitados. Evidente para ellos, la definición de los íntimos que quieren reunir puede parecer bastante flexible si se trata de objetivarla mediante algunos indicadores. Charlotte valora cosas diferentes de acuerdo con cada persona considerada. Una interacción importante o contactos regulares, por ejemplo, no le parece indispensable para que alguien forma parte de los elegidos. Entre los invitados al matrimonio así se incluyen amigos de Bruno con quienes los sus vínculos son algo débiles ${ }^{13}$. ("De hecho, es verdad que yo no me dedico a telefonear a mis amigos forzosamente una vez por semana, o ni siquiera todas las semanas. Tengo amigos a los que no veo durante 4 años, incluso a los scouts no los he visto durante 9 años..."). El matrimonio le ofrece la oportunidad de volver a relacionarse con estas personas, de recordarles una amistad que, por ese mismo acto, es proyectada de nuevo hacia un porvenir posible, a pesar de los olvidos del pasado. ("Los he visto poco antes del matrimonio, y he comprobado que por eso no he perdido la relación con ellos. Por eso los he invitado y renovado la amistad gracias a ello. He vuelto a París con ocasión de mi boda. Había dejado la región parisiense hace unos diez años, y renové el contacto en ese mismo momento. De pronto, hay algunos que he

\footnotetext{
${ }^{13}$ La pareja Cha-Bruno no figura en los cuadros estadísticos presentados en este artículo como la mayor parte de las "grandes" bodas donde los invitados no quieren enumerar los 300 invitados a la recepción, siguiendo el formato impuesto en la metodología de esta investigación.
} 
visto y nos han invitado a su casa un fin de semana, quizás fue eso lo que permitió que se renovara la relación. Así se revive una amistad, se le da un valor especial.")

Estas dudas no se refieren sino a una sola persona, que ya no es verdaderamente un amigo, y que la novia invita a pesar de ello. En esta ocasión, ignora las limitaciones financieras, probando claramente que no son las únicas que determinan la selección.

Christelle: Finalmente, me dije, ‘Oh, uno más o uno menos no será una gran diferencia'. Sí, eso (la lista) se hizo rápido. Solo por Laurent dudé un poco, un compañero de $\mathrm{IUT}^{14}$, que no veía desde hacía mucho. $Y$ después, cuando nos vimos, me sentí culpable por no haberlo invitado.

Sebastian: Y al final, ella lo invitó.

Al poner en el mismo plano amigos actuales y del pasado, Christelle abre la posibilidad de reanudar relaciones que no está dispuesta a abandonar definitivamente y que así espera reactivar.

Gaelle (asistente de comunicación) también narró un caso de conciencia en relación con una compañera de la universidad muy buena, a la que perdió de vista y no volvió a ver durante cinco años salvo en raras ocasiones. Ante su relativa indiferencia en una fiesta de San Silvestre, Gaelle decidió no invitarla pero la previno con una pequeña tarjeta que tuvo una calurosa acogida. Gaelle: "Yo me dije que era una tontería, debí haberla invitado. Y mi madre me dijo: 'Ves, bastó que le dijeras que te ibas a casar para que sintiera renacer su interés'. $Y$ a partir de ese momento he tenido dudas [sobre la decisión de no invitarla] durante semanas y semanas y no nos volvimos a ver. Es ridículo, pero ella fue una persona muy importante para mí en una época".

El debilitamiento de la relación amical es sancionado con una no-invitación, de la que Gaëlle se arrepiente rápidamente al ver el renovado interés de su amiga de, quien ve en la invitación una nueva oportunidad para conservar esta amistad. En este caso, aquello que la invitación pone de manifiesto acerca del vínculo resulta inseparable de aquello que abre para el futuro.

14 IUT Instuto Tecnico Unicersitario. Nota del traductor. 
La invitación es una muestra de amistad que también puede verse como un crédito para estrechar vínculos y desarrollar relaciones nacientes. Sin duda, es necesario reconsiderar de esta manera el número significativo de personas conocidas recientemente (Tabla 3) e invitadas al matrimonio. Una figura típica de esta tendencia es el "amigo de los amigos" a quien la invitación permite manifestar de manera directa (esta vez sin intermediarios) el interés que verdaderamente se siente por él.

La invitación al matrimonio también permite afirmar la cordialidad de la amistad en relaciones formadas en el mundo profesional. Los colegas tienen una presencia muy escasa en las bodas de los jóvenes interrogados que, de hecho, están integrados desde hace poco tiempo al mundo del trabajo ${ }^{15}$. La mayor parte justifica esta selección por una clara división entre un mundo privado y un mundo público, otra manera de oponer un universo relacional escogido por ellos a un ambiente profesional impuesto. La figura del amigo y la del colega no tienen las mismas características, en particular en el momento de ingresar a la vida activa (Bidart, Pellisier, 2002). Invitar a colegas a la propia boda permite no sólo romper con esta visión dividida de los mundos sino que sirve para indicar claramente a las personas escogidas la distinción que se les ha otorgado y que las separa del resto de simples colegas.

\begin{tabular}{|c|c|c|c|c|}
\hline $\begin{array}{c}\text { Nombre de } \\
\text { la pareja }\end{array}$ & Max & Min & Med & N amigos \\
\hline cat ala & 9 & 7 & 8 & 2 \\
\hline sop vin & 1 & 1 & 1 & 2 \\
\hline sid chr & 20 & 1 & 9 & 9 \\
\hline nat thi & 21 & 0 & 6,5 & 14 \\
\hline mar cyr & 23 & 3 & 11 & 17 \\
\hline san ste & 19 & 1 & 6 & 17 \\
\hline flo fab & 22 & 0 & 8 & 19 \\
\hline ell emm & 20 & 0 & 9 & 21 \\
\hline del pas & 25 & 3 & 7,5 & 22 \\
\hline val ben & 25 & 0 & 8,5 & 29 \\
\hline gae pie & 29 & 0 & 5 & 29 \\
\hline cel thi & 26 & 1 & 6 & 30 \\
\hline mar ced & 31 & 2 & 8,5 & 30 \\
\hline chr seb & 29 & 2 & 10,5 & 47 \\
\hline
\end{tabular}

\footnotetext{
${ }^{15}$ Según se seleccione la población estudiada, las parejas interrogadas estarán en una fase más o menos similar del ciclo de vida, lo que hará comparables sus experiencias de vida. Su juventud permite limitar en gran medida el número de estratos de su sociabilidad, especialmente aquellos relacionados con los eventuales cambios de su vida profesional.
} 


\begin{tabular}{|c|c|c|c|c|}
\hline noe vin & 16 & 0 & 6,5 & 49 \\
\hline Medias & 21,1 & 1,4 & 7,4 & 22 \\
\hline
\end{tabular}

Tabla 3. Antigüedad de los vínculos con todos los amigos invitados a la boda.

Este tipo de situaciones indica claramente cuán difícil es separar, en las relaciones de amistad, aquello que se vincula con el pasado o con él futuro, con la causa o con la consecuencia, donde algunos signos de amistad son a la vez expresión de la relación tanto como condición de su prolongación.

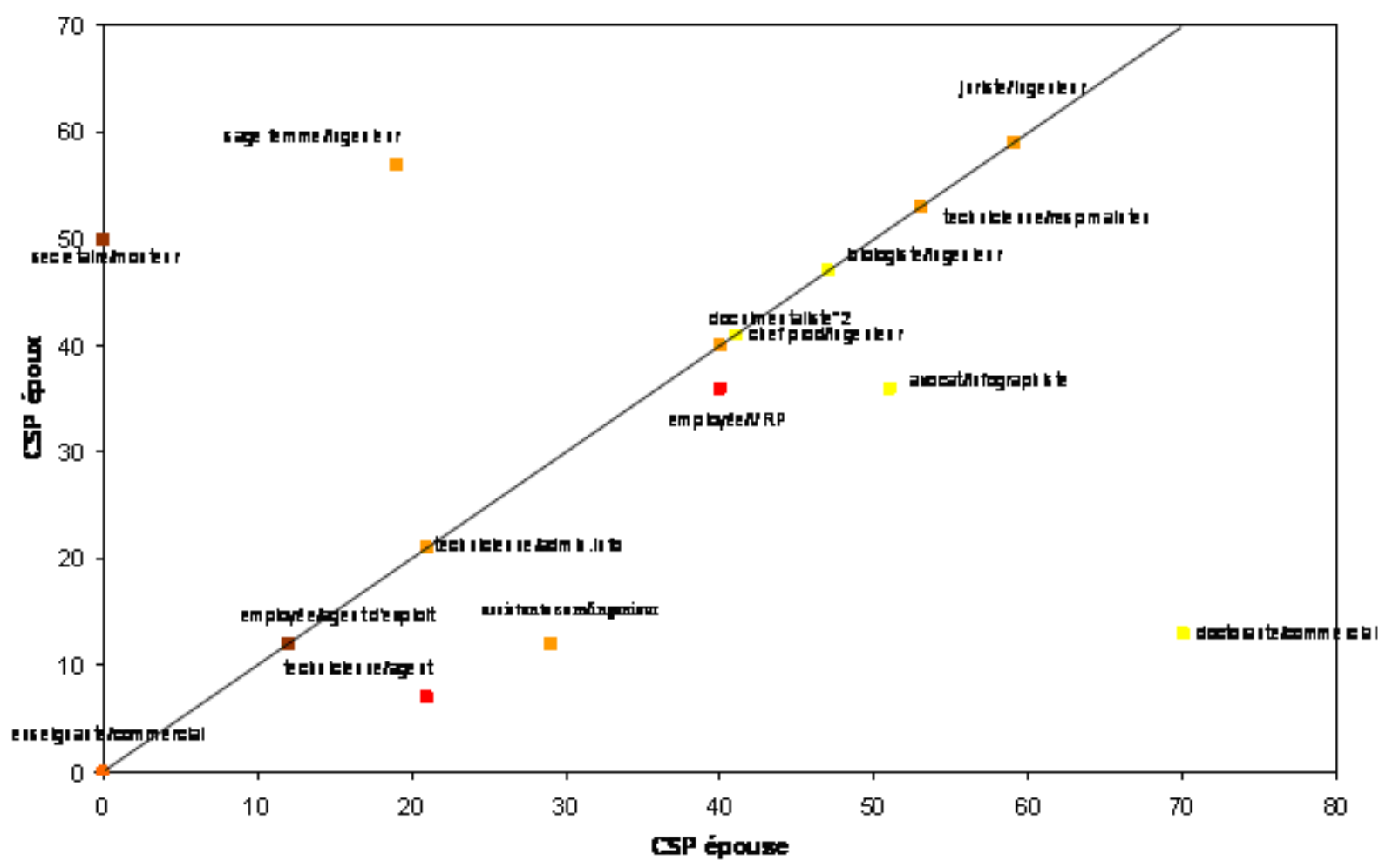

Figure 1. Amigos de la pareja con la misma profesión.

Leyenda: Cada punto representa una pareja (nombrada a partir de las ocupaciones de los cónyuges) distribuida sobre el \% de amigos de la misma CSP que la esposa (abcisa) y la misma CSP que el esposo (ordenada).

\subsection{De la “relación” al "vínculo": la fuerza creadora de la invitación}

Si la invitación al matrimonio pone de manifiesto la fuerza de una amistad, pasada o futura, aún es necesario - para que este acto tenga sus efectos- que sea percibido y reconocido como tal, es decir, como el signo distintivo de un vínculo, expresado solemnemente por la situación específica de la boda.

Para Bruno (ingeniero), invitar a los scouts, que no ha visto desde hace 9 años, se legitima por el interés que ellos manifiestan por la boda y por la respuesta positiva que le otorgan. El se arrepentirá de su acto sólo por uno de ellos que, a lo largo de 
toda la ceremonia, no testimonia más que un entusiasmo moderado por la institución matrimonial en general y por la unión de Charlotte y Bruno en particular. “Y además él tenía el aire de decir: 'Ya está hecho, mi pobre, te has ido, no hay nada que hacer, no vas a poder gozar de la vida'"... de este modo, Bruno se siente "decepcionado por su comportamiento" y duda de la posibilidad de continuar la relación.

Aunque la invitación, en cierta manera, otorga el derecho a formar parte de los happy few, no deja de implicar por eso ciertos deberes: en primer lugar, dar una acogida positiva a esta invitación. De la misma manera en que invitar (o no invitar) es portador de un sentido para cada una de las relaciones, aceptar (o no aceptar) la invitación es también un signo que los esposos decodificarán e interpretarán a la luz de la relación que cada invitado tiene con ellos.

La mayor parte de los esposos son particularmente sensibles al entusiasmo que su invitación puede suscitar y a los esfuerzos que los invitados realicen por ellos. Numerosos son los que resaltan la alegría que sienten al ver "toda esa gente reunida sólo por nosotros". Más allá del placer, muy comprensible, de que sean numerosos los presentes en la fiesta, sobre todo en la medida en que ha sido preparada con esmero, constituye también un reconocimiento de la pareja y de cada uno de los participantes.

Christelle y Sebastián insisten en como la intimidad que comparten con cada uno de los invitados es relativa y reevaluada en función de la importancia que éstos otorgan a la muestra de amistad que se les hace a través de la invitación. Para Christelle la invitación es claramente la expresión (inscripción y llamado simbólico) de una fuerte amistad. (Christelle: "Si nuestros amigos que vemos todas las semanas o más o menos cada quince días no hubieran estado allí, no hubiera sido lo mismo...") que proporciona incidentalmente la ocasión de sentirla y ponerla a prueba. Christelle analiza también las respuestas a su invitación: "Yo creo que es allí se ve... Porque justamente nos casamos en el mes de agosto y se vió quienes cambiaron la fecha de sus vacaciones y quienes no lo hicieron".

Aceptar la invitación es declararse dispuesto a celebrar, pero es también, de cierta manera, poner el sello a una relación mostrando que se acepta consagrar la atención, el tiempo y, muy a menudo, un presupuesto importante a la pareja y a la organización de su boda. En ciertos casos, estos esfuerzos proporcionan de alguna manera la nueva simiente de la intensidad de la relación, incluso cuando esta ha estado un poco debilitada. A propósito de aquellos amigos que veían con menor 
frecuencia, Christelle señala, por ejemplo, el placer que tuvo al constatar el cuidado que tuvieron en hacer honor a la invitación que se les había hecho.

Christelle: “Oh, sí, era necesario que estuvieran allí. Su presencia solamente... Natalie es de Marsella ${ }^{16}$ Es parte del grupo de los que tuvo que conducir mucho para venir. Lo mismo que Isabelle... Además. Las chicas habían ido a la peluquería, se hicieron moños, fue súper simpático. Se habían preparado y todo. Era lindo. Se sentía que estaban felices de estar allí ellas también."

La descripción de Christelle está llena de otros ejemplos de relaciones débiles o poco importantes con personas que aceptaron la invitación con placer. Se trataba de una familia con la que había hecho un intercambio en su juventud. ("Cuando vi mi familia de Inglaterra, llegaban de Gibraltar, habían venido en avión. Cuando los vi, se me partió el corazón. Cuando salí de la iglesia, estaban al final de la nave, yo los vi, me hicieron un guiño y... Son personas a las que aprecio mucho y que estuvieran allí..., jamás pensé que vendrían...") y por otra parte los vecinos de la infancia ("Los vecinos que había invitado... Mis padres vivían en una pequeña aldea e invité a todos los vecinos de B.ville, que mis padres habían abandonado hacía cinco o seis años... Con ellos había pasado mi infancia... viví allí unos quince años. Eran vecinos que aprecio mucho. Había invitado a ocho y los ocho estaban allí. Fue muy conmovedor. Todos estuvieron para el vino de honor. Uno de ellos incluso había postergado sus vacaciones para poder venir, únicamente al vino de honor y a la iglesia").

El interés despertado entre los convidados, así como su compromiso y sus esfuerzos, son apreciados como atenciones por los novios, confirmándoles la buena elección de sus invitaciones. Hasta el punto de compensar a veces la debilidad de la relación con personas invitadas por conveniencia, como es el caso de los conocidos y los empleados del comercio de los padres de Christelle que invitó en el último momento para llenar una mesa donde se había producido una ausencia repentina, y de cuya presencia después se felicita.

Christelle: Y lo pasamos súper bien. Eran gente adorable.

\footnotetext{
${ }^{16}$ La boda de Christelle tuvo lugar en Caen. La distancia entre Marsella y Caen es de más de 1000 kilómetros. Nota del traductor.
} 
Sebastien: Estuvo bien que se quedaran también para la comida, porque cantaron un poco... y no estuvo nada mal.

Christelle: Estuvo muy, muy bien. Hicieron para nosotros dos o tres cosas muy simpáticas.

Aceptar una invitación equivale a reconfirmar la confirmación que, a través de la invitación, se ha hecho de la importancia de una relación. El entusiasmo manifestado por este evento asegura que la relación así creada se estrecha.

El carácter codificado y estructurado de la boda que se manifiesta a través de su lista de invitados fijada con mucho tiempo de anticipación, sus tarjetas de comunicación del matrimonio o de invitación, sus listas de regalos donde también se objetiva "quién da qué" contribuyen también a formalizar las relaciones entre los invitados y los esposos. Estar presente parte de la boda es formar parte de uno de los episodios memorables de la historia conyugal y familiar que está por escribirse. Contrariamente a otros festejos (aniversarios, inauguración de una casa, etc.) donde la lista de invitados es menos estricta en cuanto al cupo y menos controlada, formar parte de la boda constituye una especie de sello que marca la relación de manera duradera. Al penetrar en el cuadro institucional del matrimonio, que proporciona una ocasión festiva de mezclar familias y amigos, las relaciones informales de los amigos son en cierta manera promovidas casi al rango de "vínculos" familiares, si se extiende a la amistad la definición que Claire Bidart y Anne Pellissier (2007) proponen en su análisis de las relaciones familiares. En efecto, las autoras distinguen la dimensión formal, institucional y estructural del "vínculo" de su expresión afectiva, variable según las situaciones, que ellas llaman "relaciones"17. No se trata de fundir los tipos, familiares y amicales, sino de mostrar que la invitación al matrimonio, puesto que brinda una ocasión poco común de explicitar y formalizar una relación de amigos, le confiere a ésta una condición intangible e inalienable que la aproxima al "vínculo". Quizás se debe interpretar en este sentido la idea de que la participación a una boda instaura de alguna manera una forma de pertenencia a la familia. No se trata sólo de extender el círculo de la alianza mediante la aproximación de dos familias, sino de establecer en la relación amical algo más estable y codificado que los signos informales y de renovar

17 Es posible distinguir "dos modalidades de relación intergeneracional: el vínculo, que designa el lugar y el papel simbólicos, antropológicos y estructurales de los padres y de sus hijos, y la relación; es decir, la manera coyuntural de vivir la paternidad y la manera de interactuar los padres y los hijos" (Bidart, Pellissier, 2007). 
aquellos sobre los que, por lo general, se fundan y extraen su fuerza las relaciones de amistad (Bidart, 1997), puesto que nada, en principio, supone una obligación.

La celebración conduce incidentalmente a una evaluación. Proporciona la oportunidad de contar a los amigos y de recordarles cuán importantes son ellos para la pareja, tanto respecto al pasado como al porvenir. En esta operación de selección no es fácil distinguir aquello que prueba la relación con cada uno de los invitados de aquello que la crea. La invitación al matrimonio reinscribe el pasado de la "relación" y permite diseñar su futuro proyectándolo como un "vínculo" posible. No se trata solamente de la temporalidad de las relaciones sino de su misma naturaleza, que así queda comprometida. Establecer así la lista de los amigos invitados es pues un acto de revelación y también de proyección que, más allá del porvenir de la relación misma, tiene significado y alcance sociales.

\section{La red social: la invitación como expresión de una trayectoria y de una proyección social}

\section{1. "No invitar" o la instauración de una "distinción"}

La invitación (o no) a un matrimonio no es un acto neutro para la historia de una relación. "Ser invitado" promueve a la persona al círculo de los íntimos de los novios. "No ser invitado" la excluye, de hecho, de dicho grupo. Aunque el límite sea relativo (el valor de la relación inclusión/exclusión difiere según el número total de invitados a la boda) y arbitrario como toda división ${ }^{18}$ ("Tarde o temprano, es preciso reducir la lista", nos recuerda Bruno), este límite merece ser interpretado. También los novios en sus elecciones tratan generalmente de no desilusionar a las personas que pueden esperar una invitación justificándose con razones prácticas. Es decir, reafirmando el carácter no afectivo de las razones de su eleccion. Muy a menudo, las razones económicas justifican de manera tangible y necesaria el carácter arbitrario, e incluso injusto y doloroso, de esa selección. Se debe evitar "hacer daño" como lo sugiere Charlotte, o "entristecer" como lo enuncia Noémie, quienes utilizan esas razones para incluir personas que no pertenecen al círculo de los “íntimos".

Al igual que no invitar, declinar la invitación es un acto que puede poner en peligro la relación y su significado. Para quedar bien, Sebastián (responsable de compras)

\footnotetext{
${ }^{18}$ Como en la lista de los laureados en los concursos analizados por Bourdieu (1982), la diferencia entre el último incluido y el primer excluido es siempre tenue, pero establece una barrera simbólica, ineludible e irreversible.
} 
tiene que encontrar una justificación para la ausencia de un colega con el que contaba. La encuentra finalmente en la relativa debilidad de la relación, que expresa así: "Pero si no es un amigo muy íntimo, es un amigo compañero de trabajo". Aun cuando los invitados también tengan buenas razones para no concurrir a la boda, su negativa no debe poder interpretarse en términos relacionales. Como las no invitaciones, las ausencias siempre deben estar seriamente justificadas para no suscitar dudas respecto del interés que se presta al matrimonio y en consecuencia, a la relación con la/s persona/s que invitan. Para evitar todo malentendido respecto de la relación, es necesario dar una justificación por la ausencia. Siendo por lo general profesionales, familiares o de salud, las razones personales tienen a menudo mala acogida, mientras las económicas se presentan infrecuentemente (como si supusieran fijar un costo a la amistad); aunque estas razones son muy reales y los novios las utilizan a menudo para limitar el número de invitados.

Pese a que a muchos novios no les gusta hacerlo, el recurso a la lista obliga, de hecho, a poner un límite, una frontera, que excluye a determinado número de personas. Aun cuando les den más importancia a las personas que agregan, la mayoría de las parejas tienen también personas para descartar. Así, Marion (responsable de logística) enumera todas aquellas personas que desea reunir antes de recordar una serie de personas a las que quisiera excluir a cualquier precio.

Marion: Hay personas a las que no queremos invitar, para nosotros esto es algo normal. No es necesario tomar una decisión. ' ¡Vaya!, a estos no los invitamos? A los otros era obvio que no los hubiéramos invitado. No dijimos '¿Y con ellos qué hacemos? ¿Los invitamos?'. Era claro que hay personas que queremos ver en nuestra boda y otras que pensábamos que no debían estar allí.

Los casos de exclusión de amigos son menos frecuentes que los de miembros de la familia, quizás porque son menos fáciles de descubrir para el sociólogo: la ausencia de un amigo se revela con menor facilidad que la de un padre o de una madre. No obstante, la descripción de los amigos que no han sido invitados o, en un sentido más amplio, de las personas "litigiosas" - respecto de las cuales los novios no saben si deben invitar o no- permite desvelar un cierto número de estrategias aplicadas en el proceso de selección de los invitados.

Muy pocos de los relatos ponen en evidencia las desavenencias que de repente cuestionan una invitación. Christelle de Caen relata, empero, la historia de dos 
invitaciones "problemáticas" que tuvieron objeciones a pesar de que las había propuesto con toda seguridad. La primera historia dolorosa se refiere a una amiga parisina que había elegido como testigo (vacilando de todos modos entre ella y una amiga de Caen a quien finalmente escogió) y a la que renunció a incluir entre los invitados. Aunque Christelle estaba dispuesta a pagarle el tren Paris-Caen para que fuera a aconsejarla sobre el vestido de novia, la amiga en cuestión terminó por fallarle a último momento. "Y el día antes de viajar, me llamó y me dijo: 'Oh, tengo otra cosa que hacer'. Yo lo tomé a mal. Yo lo tomé a mal porque no se le dice a todo el mundo que venga a escoger el vestido de novia y, además, la víspera de venir... Ella me dijo: 'Yo te vuelvo a llamar'. Nunca me llamó. Se trata de alguien muy individualista, Cuando ella me hizo eso, no me sorprendió demasiado, porque se trata de una persona que, bueno... Ella tenía otra cosa mejor que hacer que ir a elegir el vestido de novia. Pero bueno, yo lo tomé a mal". El disgusto es irreparable; la amiga llamó al día siguiente y Christelle nunca le envió la tarjeta de invitación.

La segunda historia se refiere al rechazo de la invitación por parte de una amiga de mucho tiempo. Aunque la amiga hizo el esfuerzo, siendo panadera, de pedir un día de licencia el sábado para ir al matrimonio, de pronto se sintió molesta por la consigna sin excepciones que habían fijado los esposos: "Nada de niños en la cena".

Sebastien: Ella no nos llamó. Nos envió un fax diciendo concretamente, textualmente: 'De ninguna manera nos desharemos de nuestros hijos para asistir a una ceremonia. Festejen sin nosotros. Nos verán en el vino de honor'.Así, de una manera bastante dura....

Fuera de estos ejemplos, son raros los disgustos afectivos que llevan a desistir de una invitación al matrimonio. Los novios invocan motivos prácticos o técnicos, antes que de relación, para no invitar a la ceremonia. Uno de ellos es prever dificultades para el desplazamiento. Elise (partera) y Emmanuel (ingeniero) en París, por ejemplo, tuvieron dudas acerca de un amigo del novio que era militar, y finalmente lo excluyeron suponiendo que estaba en Bosnia. Delphine (jurista) renunció a invitar a una amiga de la infancia que vivía en el suroeste, considerándolo muy distante de Paris, etc. Christophe y Sidonie (documentalistas de Caen) se refieren también al mismo tipo de exclusión de la lista. 
Christophe: De hecho, desde el principio, se dejaron de lado cuatro personas, Dominique, Karine, Pierre y Myriam, de Estrasburgo19, el problema se resolvió rápido.

Sidonie: Ah, sí. Eran gente que vivía muy lejos. Quizás habrían venido si se hubiesen dicho: 'No conocemos la región, vamos a pasar allá algunos días' pero, al mismo tiempo, eso podía ocurrir con algunos que hacía uno o dos años que no veíamos, pero para otros, que llevaban más de cuatro años sin vernos... En mi opinión, no se justificaba invitarlos.

Aunque la distancia geográfica puede ser considerada como una limitación, a veces tiene aspectos positivos que los esposos pueden aprovechar. Los novios a veces deciden celebrar la boda en lugares alejados (con frecuencia el lugar de residencia de los padres) por razones económicas o estéticas, pues eso les permite descartar a una cierta cantidad de personas que viven cerca de su lugar de residencia (Maillochon, 2002).

\subsection{La distancia social y los secretos de la invitación}

Aunque los esposos señalan con frecuencia el alejamiento geográfico como motivo para explicar la distinción entre los invitados y los no invitados, la lógica del argumento merece un análisis más amplio. Si el alejamiento puede justificar la exclusión, la inclusión no siempre es el resultado de una proximidad física; más bien, la verdad es lo contrario. Al igual que en el caso de la sociabilidad (Fischer, 1982; Wellman, 1997: Grosetti, 2005), invitados que llegan de los cuatro extremos de Francia, a veces del mundo entero, están presentes en casi todos los matrimonios, en particular en los más grandes en cuanto al numero de invitados.

La lejanía no es forzosamente un obstáculo para la invitación ni para su aceptación. Por el contrario, Christelle de Caen puede enorgullecerse de su amiga de Marsella y de la familia que la recibió en Londres proveniente de Gibraltar. Elisa y Emmanuel se regocijan al pensar en una pareja que viene desde Alemania. Noémie y Vincent, de amigos llegados de USA y de Israel. Camille y Matthieu de los amigos provenientes de Canadá, de Bélgica, de Bali, mientras otros, desdichadamente, no llegan de Australia, etc. Desde este punto de vista, el alejamiento geográfico podria funcionar más para las parejas, como una razón para la exclusión a priori, que para los invitados, para su propia exclusión a posteriori.

19 La distancia entre París y Estrasburgo es de más de 480 kilómetros. Nota del Traductor. 
Al analizar en profundidad los motivos de la exclusión geográfica, encontraremos otros argumentos más sociales20. La distancia geográfica es un obstáculo sólo en presencia de una proximidad afectiva21 o social que la atenúa. Por el contrario, pareciera que la distancia geográfica sirviera de elemento de rechazo cuando es reforzada por una distancia afectiva, aunque también social, como puede verse en los casos de rechazo después de dudas. En el caso del amigo militar de Emmanuel (ingeniero), se corría el riesgo de que su esposa asistiera sola, puesto que "es una persona muy simple, no trabaja. Se queda en la casa... No es un defecto, pero en todo caso..." El amigo de Pascal (ingeniero) es una persona "cuyos caminos han tomado una dirección diferente". Recíprocamente, aquellos que asisten a pesar de la distancia generalmente pertenecen a categorías superiores en la escala social. Son los invitados mejor situados económicamente, y también culturalmente, que pueden permitirse tales desplazamientos y cuya presencia es bienvenida afectiva y socialmente. Para Noémie (abogada), Camille y Mathieu (managers, diplomados de una 'Grande Ecole'), se trata de amigos del "mismo mundo": ingenieros, managers o juristas que están en su primer empleo o realizan en el extranjero una pasantía para diplomarse. En cuanto a las parejas menos favorecidas, se trata de un mundo cuyo capital parece dar prestigio a los esposos. Valerie y Benoit, de condicion modesta, hablan con satisfacción de un amigo diplomático que les hizo el honor de trasladarse hasta la región parisina; Bérangère (enfermera en hospital) y Fabien (jefe administrativo) se refieren a un actor que viene desde el Sur hasta París.

Las figuras sociales de este tipo dan brillo a la mayor parte de las bodas, reforzando la importancia de la homogeneidad social que se encuentra en la mayoría de los matrimonios de las clases medias o superiores. $O$ de la respetabilidad social en los más modestos. En efecto, el análisis sistemático de las profesiones de los invitados (Gráfico 1) muestra una fuerte homofilia social, que se acentúa en la medida en que se asciende en la jerarquía social (y el tamaño de la celebracion)22. Una tercera parte de los amigos invitados pertenecían a la misma categoría socio profesional del esposo o la esposa. En la mitad de las parejas la

\footnotetext{
20 Los numerosos trabajos que tratan de definir la "proximidad" esencial que caracteriza la relación de amistad señalan sobre todo la dificultad de separar cada una de las dimensiones según las cuales es posible evaluarla (Maillochon, 1999).

${ }^{21}$ Así pueden interpretarse también los trabajos que muestran la "superficie social" variable de la red según su contenido afectivo (Bidart, Fribourg, 2004).

22 No hay una proporcionalidad estricta entre los ingresos de las parejas (y las familias) y la cantidad de invitados. De todos modos, los matrimonios observados con más invitados de la muestra son de hecho son los más adinerados (Maillochon, 2008a).
} 
proporción supera incluso el $40 \%$. Parece observarse una cierta inercia social en la elección de los amigos convidados a la boda.

La cuestión de la distancia social pareciera poder aclarar también las decisiones tomadas en los casos dudosos donde está en juego el porvenir de la relación. En el caso del desacuerdo afectivo entre Christelle y su amiga panadera, verosímilmente hay algo del orden de la distinción social. La continuación de esta entrevista expone en todos los tonos como es esta muchacha "del campo". "No es como nosotros que somos más de la ciudad (suburbio de Caen)", agrega Christelle, que concluye: "es otro mundo". De manera recíproca, la amiga que la reemplaza es un muchacha de "La Ciudad" (Paris) y estudiante de Bellas Artes, con la promesa de un bello porvenir. En efecto, es la proximidad social la que parece operar en los casos de las invitaciones que, finalmente, son hechas aunque no se las había previsto ni deseado invitar especialmente. En los grupos que Bruno no quiere dividir (sus compañeros de apartamento23 y sus compañeros scouts) o incluso aquellos a los que Noémie se niega a diferenciar, se encuentra una fuerte homogeneidad social. A pesar de la debilidad de estos lazos directos con la pareja, estas personas son, sin embargo, invitados porque, de todos modos, forman parte "del mismo mundo".

Como lo han mostrado los ejemplos de casos dudosos, la distancia social entre los esposos y los invitados a menudo resulta decisiva para una invitación, incluso si la exclusión es justificada por otros tipos de distancia, especialmente geográfica. Por el contrario, la proximidad social permite integrar a los invitados a personas que tienen una proximidad débil con la pareja, ya sea geográfica o afectiva. La posición social de los esposos, en comparación con la de los convidados, tendría una función doble para la definición de la lista de invitados. Por una parte, la distancia social distendería las relaciones débiles y, por la otra, la proximidad social o su privilegiada posición social podrían compensar la debilidad de algunas relaciones.

La homogeneidad social, entonces, permitiría explicar de otra forma la manera en que los esposos consideran que se han rodeado de sus íntimos. Los "ineludibles" no

\footnotetext{
${ }^{23}$ Bruno : “ilnvitarlos? Sí, En realidad son grupos ya constituidos, son amigos de amigos, después invitar a un amigo de un amigo o no invitar... Porque cuando se invita a una banda, se invita a toda la banda, y ien algún momento es necesario parar!"

"En realidad no vacilamos. Yo escogí a aquellos con los que me entendía bien... bueno, los que vivían conmigo no los separé, pero entre los compañeros de apartamento, estaban los de Toulouse, yo invité a dos de ellos porque los apreciaba mucho... Después invité a la tercera. La quería evitar..."

Charlotte: En mi caso hubo dos personas, o tres, que invité porque hacían parte del grupo. Aun cuando yo no tenía especialmente mucho en común con ellos, hubiera podido ser una ocasión de hacerles daño...
} 
designan sólo un espacio afectivo sino también social, fundado en una relativa homogeneidad y también conformidad con el medio profesional de los esposos.

\subsection{El reconocimiento de la pareja a través de sus amigos y de su proyección social}

Las invitaciones enviadas para el matrimonio no sólo permiten diseñar un mapa afectivo de la red de amigos de los esposos, sino también un mapa social de las trayectorias de los esposos vistos a través de la "extensión" y de la "superficie" social de sus relaciones. La manera en que los esposos evocan, en sus testimonios, los diferentes grupos de invitados como estratos de su existencia muestra claramente este efecto de composición en torno de una trayectoria social. Los jóvenes surgidos de un medio popular son poco locuaces respecto de sus trayectorias relacionales y sociales. Por el contrario, los jóvenes que dedicaron largos años al estudio a menudo enumeran una variedad de círculos de amigos, ligados en primer lugar con sus diversas actividades escolares (por ejemplo, desde el preescolar hasta la universidad) pero también con sus distintas actividades asociativas, deportivas o festivas. Las fiestas en particular representan un escenario importante para los encuentros. Charlotte y Bruno, de este modo, se refieren así a los diferentes grupos de personas que han invitado.

Bruno: Mis amigos pertenecen a diversos grupos pues he cambiado de residencia con mucha frecuencia; estuve en la región parisina hace 10 años, estuve en ese entonces en un grupo de scouts, algunos amigos que conoci ese verano al llegar a Paris, y los he vuelto a ver a menudo, sin haber conservado el contacto, a ellos los invité. Este era un grupo de amigos. Luego están mis amigos de la escuela de agronomía, a esos los consideraremos en grupo. Después están mis amigos de la 'prepa'24, que emigraron después a los cuatro rincones de Francia, es difícil conservar los contactos... de los cuales uno es mi testigo y eso es todo. Y tú...

Charlotte: Yo tengo sobre todo los grupos de amigos de la 'prepa', porque en la 'prepa' literaria la gente se une mucho, hypokhâgnes, khâgnes25 en

Paris, y después están los amigos de la parroquia de St. Severin que los he

\footnotetext{
$24 \quad$ En Francia, para integrar la formación pública de las elites (Grandes Ecoles) se prepara un examen de ingreso durante dos años. Esta preparación es considerada como un periodo de gran prueba y marca fuertemente la trayectoria de aquellos que la siguen. Este periodo, "les classes préparatoires" es llamado "la prépa" conservaremos este nombre para hacer referencia a este periodo. Nota del traductor. $25 \quad$ Los dos prımeros años de preparación para los examenes de sección literaria reciben el nombre de hypokhâgnes para el prımero y khâgnes para el segundo. Nota del traductor.
} 
definido, pero en fin, nos conocemos por la parroquia, pero hemos hecho una cantidad de cosas incalculables juntos y por eso nos llevamos bien, formamos una buena banda de amigos. Sí, son sobre todo esos dos grupos.

Los amigos invitados son seleccionados en función de su pasado afectivo y de su posición social. Su presencia entonces representa una trayectoria histórica socialmente correcta a través de las distintas etapas de la vida afectiva de los esposos. Los invitados no narran más que el pasado social autorizado en función de la situación de los esposos en el momento de su matrimonio, o de su posible futuro, como lo testimonia Vincent (diseñador gráfico, de provincia) que no invita a todos los grupos a los que quisiera referirse sino a los que son compatibles con el nuevo universo relacional y social en el cual evolucionará en lo sucesivo, bajo la influencia de su esposa (abogada hija de padres de profesión liberal ejerciendo en París). A Vincent le hubiera gustado invitar a algunos amigos "músicos" con los cuales compartió largos años hasta el comienzo de su noviazgo, pero esta faceta de su vida social, que él quería mostrar a quienes no la conocen, no le parece compatible con las limitaciones de la realidad social que impone desde ahora su esposa. Así están también los compañeros de fútbol de Alain (responsable de ventas) que, aunque residen en los alrededores de Caen, ni son invitados ni se les informa del matrimonio: algo parecido ocurre con los compañeros de la época de fiesta de Marion que están fuera de actualidad para su óptica de hacer una boda que sea de "buen gusto".

Los amigos invitados atestiguan a la vez un anclaje social ligado a los orígenes y a su representación actual en función de aquello en lo que se han convertido o proyectan convertirse los esposos. Al igual que con la proximidad afectiva, es difícil diferenciar el registro de un pasado social de la proyección que se hace del futuro.

Al constituir un "entre-soi ${ }^{26}$ relacional", las invitaciones delimitan igualmente un "entre-soi social", aun cuando su construcción escapa muy a menudo a la conciencia de la mayoría de los esposos. Esta selección generalmente no es buscada, pero es a la vez el producto del carácter homofílico de las relaciones de los esposos y del deseo de adaptar a los invitados, tanto como a la cantidad de los mismos, al tipo de fiesta que realizan. La organización de las festividades, la elección del menú, la decoración, etc., son objeto de una dedicación particular,

\footnotetext{
${ }^{26}$ Concepto que expresa un entorno social considerado como propio en el que las personas se sienten "entre sı", protegidas y reconocidas como pertenecientes a este entorno y que comparten una cierta homogeneidad social. Nota del traductor.
} 
costosa en tiempo, en dinero, en energía, con la finalidad de lograr una "hermosa fiesta" que satisfaga a los esposos y fascine a los convidados. Ante esta doble exigencia, conviene asegurar una adecuación mínima entre los gustos de los novios y los de sus invitados que así podrán apreciar en su justo valor el cuidado especial dado a los detalles de la fiesta y eventualmente su precio. La elección de invitados, así como la atención que prestarán a la invitación, constituye una anticipación de la evaluación que harán los asistentes de la fiesta a que han sido invitados.

La relación de invitación no se puede comprender fácilmente con independencia de su dinámica constitutiva donde el pasado y el futuro de la relación se conjugan en el presente. No puede estudiarse de manera abstracta, sin integrar del mismo modo los atributos de las personas a las que permite volver a vincular.

\section{Conclusión}

La elaboración de la lista de invitados a la boda no es una operación neutra de simple mirada global de sus relaciones, tiene la función performativa de la red que permite establecer. Si la lista de invitados proporciona una especie de balance o un "fresco" relacional de la vida social de los esposos (muchos realizan esta objetivación por primera vez en su vida), también contribuye a enriquecerla y a modificarla. Al terminar la boda, las relaciones de la pareja pueden ser modificadas en función del compromiso de los invitados en la festividad (ya sea a un nivel simbólico, como una forma de compartir intensamente las emociones, o a un nivel práctico, por la ayuda logística o su participación comunicativa). Algunas proximidades pueden ser reevaluadas en función de aquello que está en juego en el desarrollo de la fiesta, ipara bien y para mal!. Pero la dimensión afectiva no parece la más importante en la selección de los invitados, aun cuando habitualmente es enunciada en primer lugar. La dimensión social es también de considerable importancia. En efecto, resulta difícil pensar en la elección de los invitados fuera de esta doble dimensión que se condiciona y se reconfigura mutuamente: la proximidad afectiva entre los esposos y los invitados no se define de la misma manera ni tiene las mismas prerrogativas según la proximidad social que los vincula. Abrir la caja negra que la relación de "invitación" constituye, describiendo la naturaleza de los vinculos que reúne así como las características individuales y sociales de las personas involucradas, permite desvelar los efectos de representación y de distinción que cada individuo pone en juego en el momento de escoger a los invitados. La relación que la "invitación" al matrimonio constituye proporciona un registro, pero también y sobre todo una proyección de la red de una pareja en el tiempo y en el espacio social. La lista de invitados no sólo nos 
suministra una descripción del brillo afectivo de la pareja, sino también de su proyección social a través de la extensión y la calidad de su red de íntimos que de este modo contribuye a crear.

A través del estudio del conjunto delimitado y objetivado de los invitados al matrimonio, aunque representa un caso muy particular de "redes sociales", es posible sugerir un cierto número de recomendaciones para hacer su análisis.

Para comprender lo que está en juego en una red de tipo ego-centrado es necesario examinar las relaciones sobre las cuales reposa bajo la forma de reciprocidad. La invitación al matrimonio no puede comprenderse sino relacionándola con la recepción de la misma. Este cambio de punto de vista, del ego al alter y del alter al ego, invita desde el comienzo a adoptar una visión más dinámica, aunque también más fenomenológica, de las relaciones que constituyen las redes y también de su dimensión preformativa. La relación - como la de invitación que sirve aquí de modelo- merece ser examinada desde una doble perspectiva dialéctica, por un lado como significado y como significante; por el otro, como el registro de un pasado indisociable de una creación del porvenir.

El análisis de la reciprocidad de la relación lleva igualmente a prestar atención a los dos individuos que ésta vuelve a vincular y sugiere su comparación. La invitación al matrimonio no puede ser analizada fuera del contexto social en el que toma forma y que la estructura, pero ésta integra también las posiciones sociales respectivas de aquellos que asocia. La elección de la invitación parece igualmente condicionada por el estatus de las personas involucradas. Este caso parece entonces sugerir la imposibilidad de pensar la relación (forma y contenido) sin tener en cuenta los atributos de las personas asociadas por ella. Frente a la amplitud de estas recomendaciones y la dificultad de aplicarlas de manera operativa, el diálogo de fuentes sobre un mismo objeto de análisis parece una posibilidad prometedora.

\section{Bibliografía}

Allan G.A. (1979). Sociology of Friendship and Family. Londres: G. Allen \& Uwin.

Bidart C. (1991). "L'amitié, les amis, leur histoire", Sociétés Contemporaines, n5, pp. $21-42$.

Bidart C. (1997). L'amitié. Un lien social. Paris. La Découverte.

Bidart C., Charbonneau J. (2007). "The contextual name generator : a good tool for the study of sociability and socialization," Communication at the XXVII Congrès de 
I'International Network for Social Network Analysis, Corfou, I nédits 2007-06, INRSUrbanisation, Culture et Société.

Bidart C., Fribourg B. (2004). «Qui sont mes proches? Proximités spatiales, proximités sociales dans les évolutions des réseaux relationnels de jeunes entrant dans l'âge adulte », 4ème Journées de la Proximité, IDEP-LEST-GREQAM, 17-18 juin.

Bidart C., Lavenu D. (2005). "Evolutions of personal networks and life events", Social Networks, vol.27, n4, pp. 359-376.

Bidart C., Pellissier A., 2002, « Copains d'école, copains de travail : évolution des modes de sociabilité d'une cohorte de jeunes », Réseaux, $n^{\circ} 115, p p .17-49$

Bidart C., Pellissier A. (2007). «Entre parents et enfants: liens et relations à l'épreuve du cheminement vers la vie adulte », Recherches et prévisions, $n^{\circ} 90$, pp.29-39.

Bourdieu P. (1982). «Les rites comme actes d'institution », Actes de la Recherche en Sciences Sociales, $n^{\circ} 43, \mathrm{pp}$. 58-63.

Bozon M. (1992)., « Sociologie du rituel du mariage », Population, n 2, pp. 409434.

Bozon M. (1991). “Le mariage : montée et déclin d'une institution " in Singly de F. (1991), La Famille : l'état des savoirs. Paris: La Découverte.

Castrén A. M., Maillochon F. (2009). «Who chooses the wedding guests, the couple or the family? Individual preferences and relational constraints in France and Finland »", European Societies, Vol. ${ }^{\circ} 11, \mathrm{n}^{\circ} 3$, pp. ${ }^{\circ} 369-389$.

Delsaut Y. (1976). «Le double mariage de Jean Célisse », Actes de la Recherche en Sciences Sociales, $n^{\circ} 4$, pp. 3-20.

Fischer C.S. (1982). To dwell among friends. Personal networks in town and City. Chicago: The University of Chicago Press.

Granovetter M.S. (1982). «The strength of weak ties. A network approach revisited" in Marsden P., Lin N. (eds), Social Structure and Network Analysis. Beverly Hills: Sage. 
Gribaudi M. (éd.), Espaces, temporalités, stratifications. Exercices sur les réseaux sociaux. Paris: Editions de l'Ecole des Hautes Etudes en Sciences Sociales.

Grossetti M. (2005). «Where do social relations come from?: A study of personal networks in the Toulouse area of France», Social Networks, 27, pp.289-300.

Héran F. (1988). «La sociabilité, une pratique culturelle », Economie et Statistique, $\mathrm{n}^{\circ} 216$, pp.3-21.

Maillochon F. (1999). «Réseaux utopiques. Formes de relations et pratiques spatiales à Paris » in Gribaudi M. (éd.), Espaces, temporalités, stratifications. Exercices sur les réseaux sociaux. Paris: Editions de l'Ecole des Hautes Etudes en Sciences Sociales, pp. 169-205.

Maillochon F. (2002). «Le coût relationnel de la robe blanche », Réseaux, $n^{\circ} 115$, p. 52-90.

Maillochon F. (2008a). «La liste des invités: une histoire d'amour, de famille et d'amitié », en cours d'évaluation.

Maillochon F. (2008b). « Le mariage est mort, vive le mariage ! Quand le rituel du mariage vient au secours de l'institution », Enfances, Familles, Générations, $n^{\circ} 9$, Faculté de droit, Université de Montréal, http://id.erudit.org/iderudit/029630ar

Maillochon, Florence (2008c). «La cérémonie de mariage: la construction d'une entre-soi familial, conjugal et social », en cours d'évaluation.

Mok D., Wellman B., with Basu R. (2007). "Did Distance Matter Before the Internet?", Social Networks, Vol. 29, n³, pp. 430-61.

Paradeise, C. (1980). "Sociabilité et culture de classe», Revue Française de Sociologie, vol. XXI, pp. 571-597.

Segalen M. (1998). Rites et rituels contemporains Paris: Nathan.

Segalen M. (1997). "Comment se marier en 1995 ? Nouveaux rituels et choix sociaux», in Bouchard G., Segalen M. (dir.) (1997), Une langue, deux cultures. Rites et symboles en France et au Québec, La découverte, Les Presses Universitaires de Laval, pp. 149-166.

Segalen M. (1998). Rites et rituels contemporains. Paris: Nathan, coll. 128. 
